\title{
Um modelo teórico para organizar e compreender as oportunidades de aprendizagem de professores para ensinar matemática
}

\section{A theoretical model for organizing and understanding teacher learning opportunities to teach mathematics}

\author{
Alessandro Jacques Ribeiro ${ }^{1}$ \\ João Pedro Mendes da Ponte ${ }^{2}$
}

\begin{abstract}
Resumo
Compreender como se constitui a aprendizagem de professores é uma importante área de pesquisa na formação de professores, com muitas questões em aberto a serem ainda investigadas. Assim, neste artigo, apresentamos um modelo teórico cujo principal objetivo é permitir o desenho de programas de formação de professores, bem como orientar a compreensão das oportunidades de aprendizagem profissional para os professores. Com base em nossos resultados de pesquisa em ensino e aprendizagem de álgebra e em uma revisão de literatura, descrevemos como o modelo foi elaborado e trazemos algumas situações ilustrativas para indicar o modelo "em ação" durante todo o processo de formação de professores. A arquitetura do programa permitiu que os professores experimentassem oportunidades de aprendizagem profissional vinculadas a conhecimentos matemáticos e didáticos sobre padrões e regularidades, bem como a oportunidade de aprender uns com os outros, superando o isolamento causado pelo trabalho diário em suas escolas e levando-os a explorar práticas próximas à própria realidade escolar.
\end{abstract}

Palavras-chave: Aprendizagem dos professores; Tarefas de aprendizagem profissional; Modelo teórico; Formação de professores.

\begin{abstract}
Understanding how teacher learning is constituted is an important research area in teacher education with many open questions to be investigated yet. Thereby, in this paper, we present a theoretical model whose main purpose is to enable the design of teacher education programs as well as to guide the understanding of professional learning opportunities for teachers. Based on our research outcomes on teaching and learning algebra and on a literature review, we describe how the model was elaborated and bring some illustrative situations to indicate the model "in action" throughout the teacher education process. The architecture of the program enabled teachers to experience professional learning opportunities linked to mathematical and didactical knowledge regarding patterns and regularities, as well as the opportunity to learn from and with each other, overcoming the isolation caused by daily work in their schools and leading them to explore practices close to their own school reality.
\end{abstract}

\footnotetext{
Submetido em: 04/04/2020 - Aceito em: 20/11/2020 - Publicado em: 04/12/2020

${ }^{1}$ Doutor em Educação Matemática pela Pontifícia Universidade Católica de São Paulo. Professor Associado no Centro de Matemática, Computação e Cognição da Universidade Federal do ABC, Brasil. E-mail: alessandro.ribeiro@ufabc.edu.br. ORCID: https://orcid.org/0000000196470274

${ }^{2}$ Doutor em Educação pela Universidade da Georgia, EUA. Professor catedrático no Instituto de Educação da Universidade de Lisboa, Portugal. E-mail: jpponte@ie.ulisboa.pt. ORCID: https://orcid.org/0000-0001-6203$\underline{7616}$
} 
DOI: $10.20396 /$ zet.v28i0.8659072

Keywords: Teacher learning; Professional learning tasks; Theoretical model; Teacher education

\section{Introdução}

Como ocorre a aprendizagem do professor? De que maneira essa aprendizagem se desenvolve ao longo de sua carreira? Essas questões são recorrentes em pesquisas que tematizam o professor, seus conhecimentos e suas práticas (e.g. Webster-Wright, 2009; Russ, Sherin, \& Sherin, 2016). Tão importante quanto as questões anteriores, são aquelas relacionadas às oportunidades de aprendizagem do professor, como, o que se entende por oportunidades de aprendizagem e como podemos propiciá-las aos professores? A ideia de “oportunidades de aprendizagem" tem sido pesquisada já há muito tempo (HeydMetzuyanim, Tabach \& Nachlieli, 2016), com ênfase em estudos envolvendo estudantes da escola básica. Na formação de professores, a busca por se compreender como se constituem oportunidades para o professor aprender é bem mais recente e tem tido como foco a formação inicial (Tatto \& Senk, 2011).

Para se compreender como se constituem as oportunidades para o professor aprender, há de se entender, primeiramente, como os professores aprendem. Neste artigo, toma-se por base, por um lado, o entendimento de que a aprendizagem do professor se situa na prática diária, incluindo os momentos de sala de aula, mas também de planejamento, avaliação e colaboração com colegas e outros (Davis \& Krajcik, 2005) e, por outro lado, que a aprendizagem do professor está distribuída entre indivíduos, bem como em artefatos, como o caso de tarefas preparadas para sua formação (Putnam \& Borko, 2000). Assim, consideramos a aprendizagem do professor como algo que

envolve o desenvolvimento e a integração de uma base de conhecimento sobre conteúdo, ensino e aprendizagem; tornando-se [o professor] capaz de aplicar esse conhecimento em tempo real para tomar decisões no ensino; participar do discurso do ensino; e tornar-se enculturado (e engajado) em uma variedade de práticas de professores (Davis \& Krajcik, 2005, p. 3).

Fundamentado nestes princípios, o framework "Oportunidades de Aprendizagem do Professor (OAP)" constitui um modelo teórico-metodológico para (i) organizar o design de processos formativos que objetivem promover aprendizagem aos professores e (ii) gerar oportunidades para os professores aprenderem durante processos formativos a partir de três domínios: (a) Papel e Ações do Formador (PAF), (b) Tarefas de Aprendizagem Profissional (TAP), e (c) Interações Discursivas entre os Participantes (IDP). As características dos diferentes componentes de cada domínio são apresentadas e discutidas mais à frente. $\mathrm{O}$ objetivo deste artigo é descrever a construção do modelo teórico OAP e ilustrar como este modelo foi utilizado para o design e a implementação de um processo formativo que tematizava o conhecimento matemático para o ensino de álgebra na educação básica. Nós discutimos ainda, se e como esse modelo pode ser usado na formação de professores de matemática em perspectiva, bem como fora da educação matemática. 
DOI: $10.20396 /$ zet.v28i0.8659072

\section{O modelo das "Oportunidades de Aprendizagem Profissional para Professores" (Modelo PLOT) ${ }^{3}$}

Para estudar se e como um processo formativo pode apoiar a aprendizagem do professor, é necessário que se organize o design deste processo para tal finalidade (Davis \& Krajcik, 2005; Fuentes \& Ma, 2017). O framework OAP (Figura 1) pode ser utilizado para identificar e avaliar o papel e as características que cada dimensão desempenha na criação de oportunidades de aprendizagem ao professor. Assim, ao se explorar a primeira potencialidade deste modelo, ser utilizado como um orientador para a organização e desenvolvimento de um processo formativo, há de se garantir que o processo formativo contemple, de forma articulada, as três dimensões.

Este artigo apresenta um detalhamento do modelo e de seus componentes, e procura fornecer elementos úteis para se balizar o design do processo formativo, o qual, em última instância, tem o intuito de promover aprendizagens que desencadeiem crescimento do professor (Loucks-Horsley, 1997; Feiman-Nemser, 2001), em especial, no que diz respeito à assunção de práticas eficazes (Lampert, 2010). Além disso, entendemos que o modelo pode ser usado como base para a constituição de um quadro de análise que permita identificar se e avaliar como um processo formativo que contemple as três dimensões (PAF, TAP e IDP) e suas características, possibilita desvelar e compreender quais foram e como se constituíram oportunidades para o professor aprofundar seu conhecimento profissional para ensinar matemática. Assim, com isso, exploramos a segunda potencialidade do modelo.

O modelo "Oportunidades de Aprendizagem do Professor" tem uma estrutura que visa romper com uma lógica linear e compartimentalizada de se conceber os processos de formação que objetivam propiciar aprendizagem ao professor (Goldsmith, Doerr \& Lewis, 2014). A adoção de uma perspectiva interativa e interconectada que considera os três domínios, inseridos em um contexto específico, em conjunto pode contribuir para gerar oportunidades de aprendizagem ao professor. Goldsmith, Doerr e Lewis (2014) depreendem de seu estudo a "necessidade de desenvolver estruturas compartilhadas para o estudo da aprendizagem dos professores" (p. 23) e, ainda sugerem que

a estrutura de Clarke e Hollingsworth (2002) pode, e talvez deva, fornecer um enquadramento comum para estudos futuros. Sua amplitude pode acomodar uma variedade de focos de pesquisa e permitir o acúmulo de descobertas que articulam ainda mais as características críticas dos domínios individuais e de suas interações (Goldsmith, Doerr \& Lewis, 2014, p. 23).

Seguindo esta sugestão, a arquitetura do modelo OAP foi desenvolvida inspirada em Clarke e Hollingsworth (2002), que apresentam em seu trabalho, um "modelo interconectado de crescimento professional” (p. 951). No entanto, o modelo OAP se diferencia deste último, uma vez que considera outros domínios como constitutivos das oportunidades de

\footnotetext{
${ }^{3}$ Optamos por manter o acrônimo PLOT da designação em inglês (Professional Learning Opportunities for Teachers) por entendemos que a sonoridade da pronúncia, mesmo em português, nos parece agradável.
} 
DOI: $10.20396 /$ zet.v28i0.8659072

aprendizagem do professor e, também, por ser um modelo que tem por preocupação buscar entender a aprendizagem e o crescimento do professor em um nível de análise mais refinado, como sugere Schoenfeld (2015).

O modelo OAP (Figura 1) foi concebido com o propósito de fornecer parâmetros para organizar e desenvolver processos formativos que visem efetivar oportunidades de aprendizagem ao professor. Por isso, além de ser composto por três dimensões, é constituído por três fases de operacionalização:

(1) Organização: momentos em que o formador elabora o processo formativo (seja no todo ou em partes) e constrói o design da(s) TAP e das potenciais IDP.

(2) Desenvolvimento: momentos em que os participantes (formador e formandos) passam a interagir entre si, mediados pelo uso da(s) TAP e pela concretização das IDP.

(3) Finalização: momento em que, por meio processo aglutinador entre as três dimensões (PAF, TAP e IDP), se efetiva a(s) OAP.

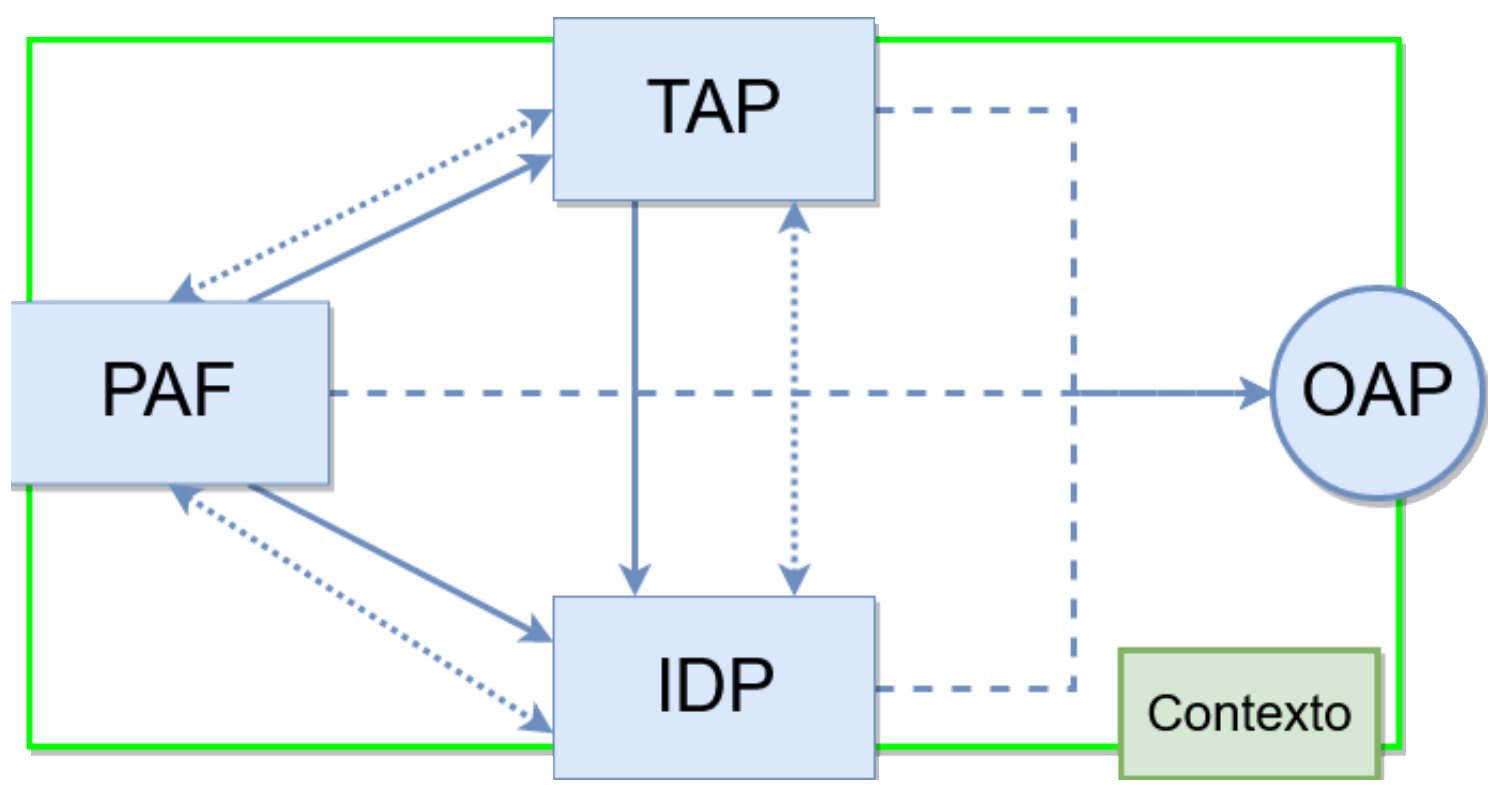

Figura 1 - Modelo “Oportunidades de Aprendizagem Profissional para Professores” (PAF: Papel e Ações do Formador; TAP: Tarefa de Aprendizagem Profissional; IDP: Interações Discursivas entre os Participantes; OAP: Oportunidades de Aprendizagem Profissional; Contexto no qual as oportunidades estão inseridas) Fonte - Elaborado pelos autores

É de notar os seguintes aspetos da Figura 1:

- os retângulos representam as três dimensões (PAF, TAP e IDP), as quais estão distribuídas de forma conectada, mas obedecendo a uma lógica de continuidade/fluidez.

- as flechas (contínuas, pontilhadas e tracejadas) indicam a continuidade/fluidez do processo e, de acordo com o sentido (direcional ou bidirecional), representam movimentos interativos entre os três domínios e que se alteram de 
DOI: $10.20396 /$ zet.v28i0.8659072

acordo com a fase de operacionalização do modelo. As fechas contínuas indicam os movimentos na fase de organização; as fechas pontilhadas, os movimentos na fase de desenvolvimento; as flechas tracejadas, ao se unirem (entre as fases de desenvolvimento e efetivação) formam uma amálgama dos diferentes domínios, que acaba por desencadear em oportunidade(s) de aprendizagem do professor.

- o círculo, representa a efetivação da criação da oportunidade de aprendizagem do professor.

- o retângulo que envolve as demais componentes - o Contexto, representa a perspectiva situada de aprendizagem que sustenta teoricamente o modelo.

\section{Considerações teóricas: princípios e fundamentos que sustentam o modelo PLOT}

Uma das principais características do modelo OAP, como discutido anteriormente, é considerar, de maneira interativa e interconectada em um único sistema, os três diferentes domínios que compõem o modelo. Ao se articular estes três domínios em um único sistema, gera-se uma ferramenta teórico-metodológica para organizar e concretizar processos formativos que fomentem oportunidades para a aprendizagem de professores que ensinam matemática.

A literatura sobre a pesquisa em formação de professores, em geral e na área da Educação Matemática, demonstra que a comunidade de pesquisadores já vem estudando os três domínios que compõem o modelo PLOT, porém, desarticulados uns dos outros. Por exemplo, o papel e as ações do formador (PAF) é estudado por Remillard e Geist (2002) e Bruce, Esmonde, Dookie e Beatty (2010). As tarefas de aprendizagem profissional (TAP) são consideradas nos estudos de Ball e Cohen (1999), Smith (2001) e Swan (2007). No que tange às interações discursivas entre os participantes (IDP), tem-se os trabalhos de Ponte e Quaresma (2016), Craig e Morgan (2015) e Nemirovsky, Dimattia, Ribeiro e Lara-Meloy (2005). Por isso, consideramos nossa proposta como uma forma nova e diferente de desenhar e desenvolver programas de formação de professores.

A ausência de integração dos três domínios, no entanto, constitui uma lacuna a superar nas pesquisas em formação de professores. Com isso, decidiu-se organizar o modelo PLOT que, como se observa na Tabela 1, possui duas dimensões (conceituais e operacionais) e quatro componentes (em cada um dos três domínios). Tais elementos caracterizam, por um lado, a estrutura e as bases teóricas (dimensão conceitual) e, por outro lado, orientam a forma pela qual o modelo é utilizado (dimensão operacional). Assim, essas duas dimensões, quando tomadas em conjunto, têm por objetivo organizar um processo de formação de professores e / ou identificar e compreender se e como surgem oportunidades de aprendizagem profissional para os professores. 
DOI: $10.20396 /$ zet.v28i0.8659072

Os Domínios do modelo "Oportunidades de Aprendizagem Profissional para Professores" (Modelo PLOT): dimensões e componentes

Papel e Ações do Formador (PAF): As componentes deste domínio são Aproximação e

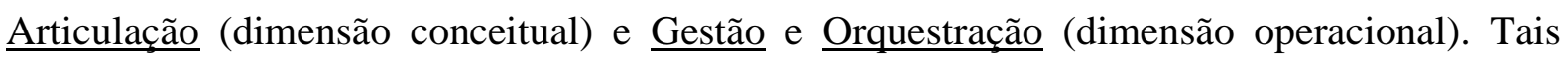
componentes levam em conta: a necessidade de aproximar a matemática acadêmica da matemática escolar (Elias, Ribeiro \& Savioli, 2019; Kilpatrick, 2019; Moreira \& David, 2008; Schubring, 2019; Wasserman, 2018); a importância de se considerar a articulação entre a matemática e a didática no e para o ensino (Ball, Thames \& Phelps, 2008; Ponte, 1999; Neubrand, 2018; Rowland, Huckstep, \& Thwaites, 2005); a relevância de se construir, por meio da gestão da "sala de aula", ambientes de ensino-aprendizagem exploratório (Jaworski \& Huang, 2014; Ponte \& Quaresma, 2016); o potencial de considerar a orquestração de discussões didáticas e matemáticas ao se pensar a aprendizagem de professores (Stein et al., 2008; Borko, Jacobs, Seago \& Mangram, 2014).

Tarefas de Aprendizagem Profissional (TAP): As componentes deste domínio são Conhecimento Profissional e Ensino Exploratório (dimensão conceitual) e Tarefa Matemática e Registros de Prática (dimensão operacional). Estas componentes emergem da relevância de se considerar: as especificidades do conhecimento profissional do professor, de modo a promover a exploração destes conhecimentos no que se refere às tarefas matemáticas que se propõem aos estudantes (Silver et al., 2007; Boston \& Smith, 2011); um ambiente de ensinoaprendizagem que favoreça a exploração e a investigação matemática (Ponte \& Quaresma, 2016; Zaslavsky \& Leikin, 2004; Jaworski \& Huang, 2014); a importância de se utilizar tarefas matemáticas de alto nível cognitivo com seus estudantes (Boston \& Smith, 2011); o papel dos registros de prática (Ball, Ben-Peretz \& Cohen, 2014) ao se compor as vignettes, por exemplo, com o uso de vídeos e seu potencial formativo (Maarten, den Hertog \& Gravemeijer, 2002; Borko et al., 2008; Coles, 2013; Beilstein, Perry \& Bates, 2017).

Interações Discursivas entre os Participantes (IDP): As componentes deste domínio são Discussões Matemáticas e Didáticas e Argumentação e Justificação (dimensão conceitual) e Linguagem Mobilizada e Comunicação Dialógica (dimensão operacional). Estas componentes são constituídas no sentido de: promover discussões matemáticas e didáticas como um meio para favorecer aprendizagem profissional aos professores (Heyd-Metzuyanim, Tabach \& Nachlieli, 2016; Ponte \& Quaresma 2016; Shilo \& Kramarski, 2018); envolver os professores em um ambiente que se promova argumentação e justificação (Mata-Pereira \& Ponte, 2017) quando se discute tarefas matemáticas para os estudantes; estimular o uso de linguagem matemática correta e adequada ao nível de ensino dos estudantes (Adler \& Ronda, 2014; Radford \& Barwell, 2016); levar os professores a reconhecer a importância da comunicação dialógica entre estes e seus estudantes (Nemirovsky, Dimattia, Ribeiro \& LaraMeloy, 2005; Craig \& Morgan, 2015).

A Tabela 1 apresenta os domínios, em suas dimensões conceitual e operacional, com as componentes e suas características, de modo a sintetizar e tornar a arquitetura do modelo OAP visível e explícita. 
DOI: $10.20396 /$ zet.v28i0.8659072

Tabela 1. Dimensões, componentes e características do modelo PLOT em seus três domínios

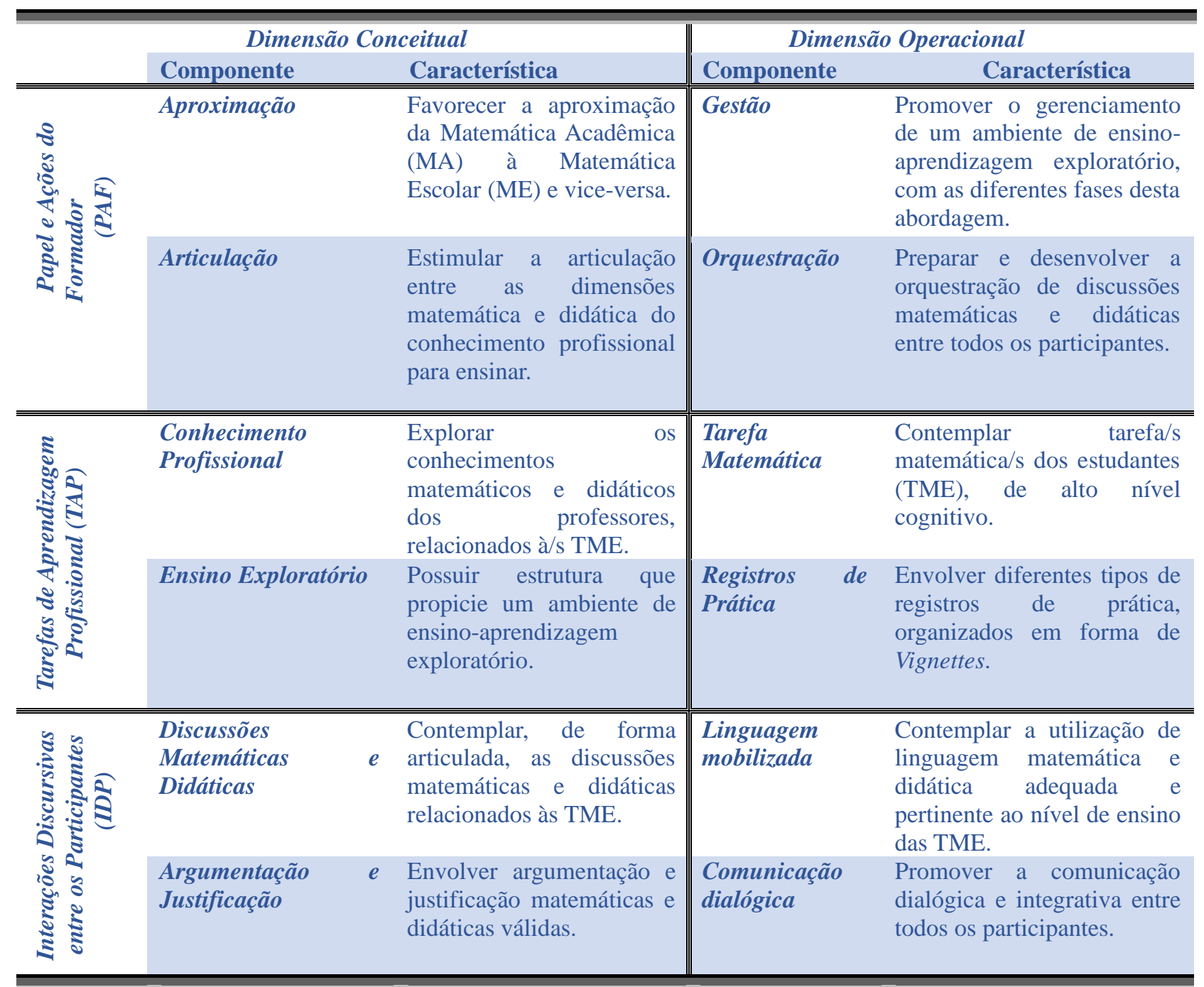

Fonte - Elaborado pelos autores

\section{Contexto do estudo: pesquisas que deram origem ao modelo PLOT}

O desenvolvimento do modelo OAP vem ocorrendo ao longo de uma agenda de pesquisa longitudinal, que toma por princípios metodológicos o Desgin-Based Research DBR (Cobb et al., 2003; Ponte et al., 2016) e, em específico, um tipo de DBR que se propõe contribuir para que professores - trabalhando em conjunto e em colaboração com pesquisadores - desenvolvam aprendizagens que lhes possibilitem seguir práticas de ensino inovadoras em suas salas de aula (Cobb, Jackson \& Dunlap, 2016).

A produção dos dados vem sendo realizada desde 2016 e os pesquisadores têm usado (i) gravações em vídeo e áudio; (ii) recolha de documentos (protocolos produzidos por alunos e professores, planos de aula, entre outros); (iii) observação de oportunidades de aprendizagem profissional (OAP); (iv) entrevistas com professores e com formadores; e (v) 
observação de aulas realizadas por professores em escolas de educação básica. Os participantes da pesquisa são (a) professores em formação inicial, (b) professores em exercício profissional em salas de aula da escola básica, (c) mestrandos e doutorandos, e (d) pesquisadores e professores universitários.

Tendo em vista a natureza iterativa e cíclica de investigações de DBR, o diagrama abaixo sintetiza as diferentes fases da agenda de pesquisa, com ênfase para aquela na qual ocorreu a recolha de dados cujo processo formativo é discutido neste artigo (Figura 2):

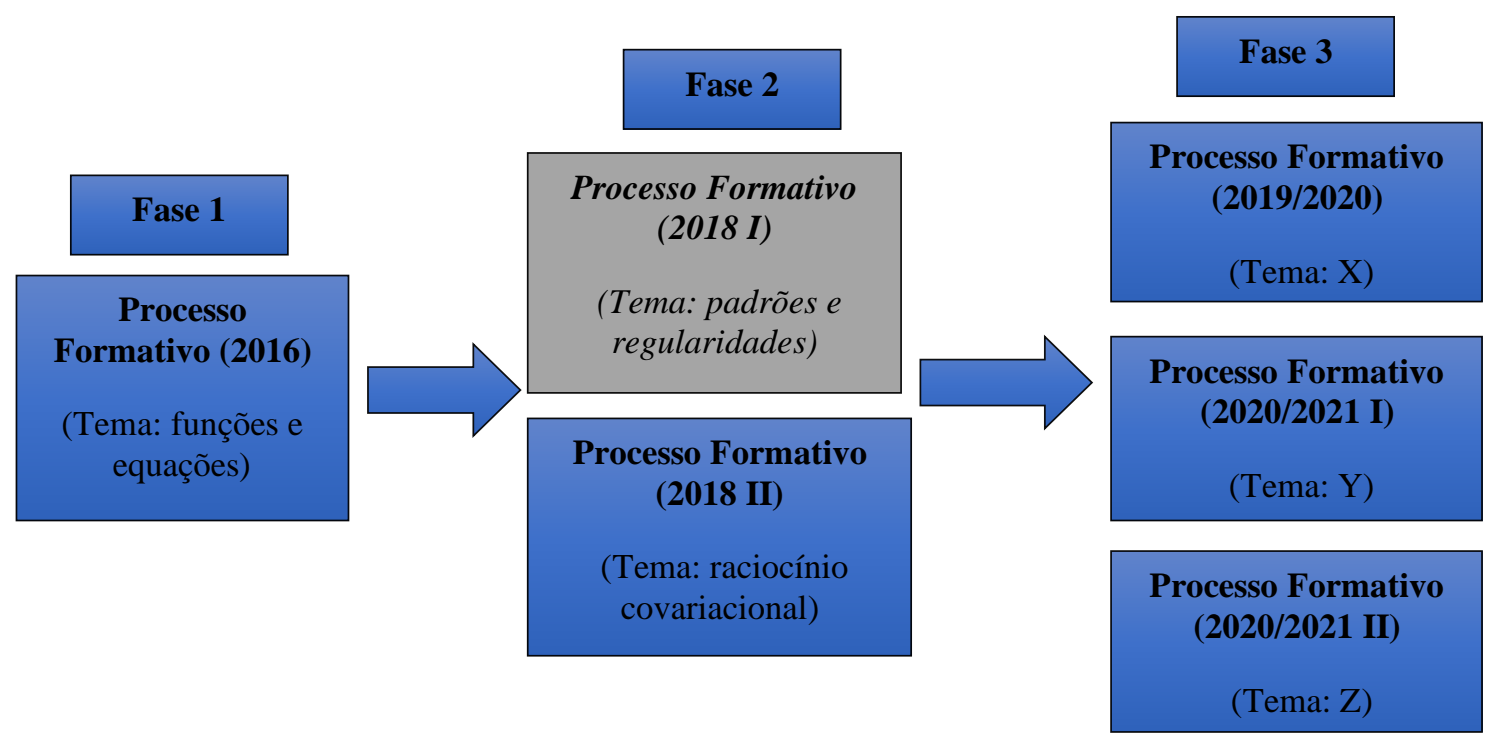

Figura 2 - Estrutura do estudo longitudinal e suas 'fases".

Fonte - Elaborado pelos autores

Assim, uma vez que o processo formativo analisado neste artigo é parte integrante da "fase 2" do DBR, como indicado na Figura 2, passamos a explicá-lo a seguir. Vale ressaltar que, na fase 3, também realizaremos estudos envolvendo a formação de futuros professores de e que ensinam matemática.

Contexto SP (Processo Formativo 2018 I): Este processo formativo foi desenvolvido ao longo de 15 encontros semanais de 4 horas. Os encontros foram dinamizados pelos pesquisadores, no papel de formadores, e conjugavam momentos de trabalhos (i) individuais, (ii) em pequenos grupos e (iii) em discussões coletivas em plenária. As sessões de trabalho contemplavam, de forma intercalada, momentos de estudo teórico (workshops, totalizando 8 horas) e momentos de trabalho do tipo "hands on", mediados pelas tarefas elaboradas pelos formadores (TAP), cinco no total; por interações discursivas entre os participantes (IDP); e pelo papel assumido pelos formadores e suas ações ao longo dos encontros (PAF). A maior parte das atividades foi realizada na universidade, tendo sido realizados três encontros em escolas de educação básica. O processo formativo contou com um total de cinco TAP desenvolvidas com o objetivo de levantar os conhecimentos matemáticos e didáticos prévios dos professores participantes sobre "Padrões e Regularidades na Álgebra Escolar" e, após encontros formativos (Workshops), outros três TAP foram realizadas com os professores. As 
TAP 3, 4 e 5 foram escolhidas uma vez que compunham um ciclo de trabalho denominado Ciclo PDR (Planejamento, Desenvolvimento e Reflexão) (Trevisan, Ribeiro \& Ponte, 2020) ${ }^{4}$, incorporando aulas elaboradas coletivamente pelo grupo de professores e possuíam o seguinte formato: (a) $3^{\text {a }}$ TAP: Preparação coletiva de planos de aula para o ensino médio, a respeito do tema padrões e regularidades, e seleção de um plano de aula a ministrar posteriormente; (b) 4 $4^{\text {a }}$ TAP: Desenvolvimento da aula selecionada em uma turma do ensino médio, por um dos professores participantes que se voluntariou para tal; (c) $5^{\mathrm{a}}$ TAP: Reflexão coletiva, mediada por registros de prática produzidos na aula desenvolvida em uma sala de aula do ensino médio. Participaram do estudo 33 professores de matemática ( 7 em formação inicial e 26 formados, sendo que dos 33 docentes, 5 não possuíam experiência em sala de aula). Para a realização das TAP os professores foram divididos em 6 grupos (4 a 6 participantes), organização feita pelos formadores de modo que, em todos os grupos, houvesse (i) professores com e sem experiência em sala de aula e (ii) professores formados e em formação inicial. A professora Jéssica, que ministrou a aula objeto de análise neste artigo, tinha 8 anos de formada e, na altura, atuava no ensino médio em uma escola da rede pública.

Na próxima seção trazemos excertos do processo formativo no intuito de exemplificar o modelo PLOT "em ação", uma vez que este fundamentou a organização e realização do processo formativo "Contexto SP". Por limitação de espaço não é possível explorar todas as três dimensões, seus domínios conceituais e operacionais e diferentes componentes. Buscamos ilustrar aspectos acerca de como o modelo OAP, de maneira geral, contribui para o design do processo formativo e, ao mesmo tempo, possibilitou que os formadores desenvolvessem a formação. Ao longo da análise buscamos identificar oportunidades de os professores aprenderem conhecimentos didáticos e matemáticos (Ponte, 1999; Ball, Thames \& Phelps, 2008) acerca de padrões e regularidades.

\section{O modelo PLOT em um processo formativo com professores de matemática}

No intuito de apontar situações ilustrativas sobre a operacionalização do modelo PLOT no processo formativo "Contexto $S P$ ", apresentamos excertos das três TAP que compõem o ciclo PDR, ao mesmo tempo em que destacamos evidências extraídas dos dados recolhidos durante o processo formativo e que nos indicam a existência de oportunidades de aprendizagem profissional (OAP) para os professores participantes. Iniciamos ilustrando, por meio de trechos das TAP 3, 4 e 5, como o modelo foi tomado para organizar o design do processo formativo, sempre articulando tais ilustrações aos componentes do modelo e, ainda, as OAP evidenciadas.

O modelo PLOT e a $3^{a}$ TAP (Planejando a aula)

\footnotetext{
4 Neste artigo os autores discutem extensivamente sobre a utilização do Ciclo PDR em um estudo com professores de matemática (em um processo de desenvolvimento profissional), sobre o conhecimento matemático e a aprendizagem relacionada ao conceito de função em seu aspecto covariacional.
} 
O encontro no qual a $3^{\text {a }}$ TAP foi abordada foi precedido pela organização, por parte dos formadores, de um roteiro elaborado para apoiar a realização do planejamento da aula pelos professores (Serrazina, 2017). Ao optarem por apresentar o roteiro para planejar uma aula no formato TAP, os formadores reconheceram seu papel de designers de tarefas formativas e, ao mesmo tempo, mobilizaram determinadas ações (PAF) em suas escolhas para criar a TAP (Figura 3). Por exemplo, a componente articulação entre as dimensões matemática e didática do conhecimento profissional (CP) levou os formadores a contemplar na TAP oportunidades para que os professores explorassem uma tarefa matemática para estudantes, tanto do ponto de vista de seus aspectos matemáticos como didáticos (considerando uma prospecção da gestão da aula futura e refletindo sobre as estratégias e dificuldades que seus estudantes poderiam ter com o envolvimento da tarefa escolhida). Ao mesmo tempo, os formadores previam potenciais IDP que deveriam surgir no desenvolvimento da TAP, uma vez que a orquestração de discussões matemáticas e didáticas (ODMD) estava prevista pelos formadores, seja pela forma como a TAP seria desenvolvida (em pequenos grupos e em planária), seja pelo próprio ambiente de ensino-exploratório previsto pelos formadores.

$1^{\circ}$ Passo - Escolham uma boa tarefa e, ao mesmo tempo, tracem as aprendizagens que desejam promover nos estudantes.

$2^{\mathbf{0}}$ Passo - A partir da tarefa escolhida e, considerando as aprendizagens que desejam promover, estabeleçam os objetivos que pretendam alcançar com a aula planejada, incluindo ainda o ano escolar e a duração da aula.

$3^{\mathbf{0}}$ Passo - Busquem antecipar as dificuldades dos estudantes e suas possíveis estratégias de resolução para a tarefa.

$4^{\circ}$ Passo - Antecipem possíveis perguntas do professor e respostas dos estudantes.

$5^{\circ}$ Passo - Definam quais recursos (materiais e didáticos) serão necessários para a realização da aula; utilizem o questionamento como apoio à aprendizagem dos estudantes; estabeleçam que os estudantes trabalhem em grupo.

$6^{\circ}$ Passo - Preparem a avaliação, a qual deverá ocorrer ao longo da aula (Lembrem-se da proposta de Stein e colaboradores (2008)).

$7^{\circ}$ Passo - Preparem a tarefa dos estudantes.

Figura 3 - Trecho da $3^{\text {a }}$ TAP com as orientações para os professores.

Fonte - Dados da pesquisa

Os planos de aula elaborados pelos professores foram apresentados, discutidos e selecionados no encontro formativo posterior, encontro este mediado pela $2^{a}$ parte da $3^{\mathrm{a}}$ TAP (que tinha por objetivo subsidiar os professores na análise da completude e da qualidade do plano de aula que seria aplicado em sala de aula). O plano de aula escolhido foi "Investigando padrões por meio do jogo Torre de Hanói".

O modelo PLOT e a $4^{a}$ TAP (Desenvolvendo a aula)

A $4^{\mathrm{a}}$ TAP previa a realização da aula planejada anteriormente, o que ocorreu na aula da professora pertencente ao grupo que elaborou o plano de aula, em uma escola pública da região metropolitana de São Paulo, em uma turma do $3^{\circ}$ ano do ensino médio. A aula teve 
duração de 150 minutos e contou com a participação de 33 alunos divididos em 3 grupos de 5 (grupos 3A, 3B e 3C) e 3 grupos de 6 (grupos 3D, 3E e 3F). Durante a aula estiveram presentes dois formadores e dois professores participantes do processo formativo, com o objetivo de filmar e observar a aula. A $4^{\text {a }}$ TAP (Figura 4) tinha por objetivos (i) guiar os professores observadores para um "olhar" mais focado e detalhado de certos elementos do desenvolvimento do plano de aula e (ii) proporcionar a produção de registros de prática (Ball, Ben-Peretz \& Cohen, 2014) que seriam utilizados, posteriormente, na $5^{\text {a }}$ TAP.

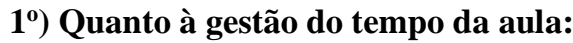

a) A aula desenvolvida permitiu a participação e a manifestação dos alunos ou priorizou a fala do professor?

b) A aula desenvolvida permitiu aos alunos compreenderem e se envolverem ao longo da atividade?

c) A aula desenvolvida permitiu discussões nos pequenos grupos e na plenária?

$\left.2^{\circ}\right)$ Quanto às ações do professor:

a) O professor explicitou as orientações necessárias e suficientes para que os alunos se envolvessem na aula?

b) O professor utilizou-se de perguntas e afirmações para ajudar os alunos a compreenderem os conceitos envolvidos na aula?

c) O professor utilizou-se de terminologia apropriada (de acordo com a Matemática e com a idade dos estudantes) e uma linguagem adequada para ajudar os alunos a fazer as conexões necessárias?

d) O professor, ao final da plenária, possibilitou sistematização dos conhecimentos matemáticos envolvidos na tarefa?

$3^{\circ}$ ) Quanto às discussões dos alunos durante a plenária:

a) O professor possibilitou que os alunos apresentassem maneiras diferentes de realização da tarefa (incluindo possíveis estratégias incorretas)?

b) O professor considerou as dificuldades apresentadas pelos alunos e fez intervenções de modo a saná-las?

c) O professor promoveu debate entre as diferentes estratégias apresentadas pelos alunos e em relação às suas dificuldades?

Figura 4 - Trecho da $4^{\mathrm{a}}$ TAP com orientações para os observadores.

Fonte - Dados da pesquisa

O roteiro elaborado para a $4^{\mathrm{a}}$ TAP ratifica o papel que os formadores assumem como designers de tarefas formativas e pode ilustrar outras ações dos formadores (PAF) para construir a TAP e escolher seus componentes. Nota-se aqui, as componentes articulação (que estimulava os observadores acerca das dimensões matemática e didática do conhecimento do professor que realizava a aula); orquestração (de modo que os observadores poderiam identificar como o professor conduzia as discussões ao longo da aula); gestão (que direciona o olhar dos observadores para a forma como a professora gerencia o ambiente de ensino exploratório previsto no plano de aula).

A $4^{\text {a }}$ TAP, ao mesmo tempo que subsidiava a observação da aula realizada pelos professores e formadores, permitia a produção e recolhimento de registros de prática para utilização posterior no processo formativo. Por exemplo, foram extraídos dois episódios no 
DOI: $10.20396 /$ zet.v28i0.8659072

desenvolvimento da aula, um deles relativo às discussões entre a professora e os estudantes sobre uma estratégia de resolução da tarefa não prevista anteriormente:

E13F (Estudante 1 do grupo 3F): Professora coloca assim $n$ igual a $n_{a}$ vezes 2 mais 1. [a professora escreve na lousa $n=n_{a} \times 2+1$ ].

Jéssica (Professora Jésica): E o gráfico de vocês ficou como? Oho! Acho que deu alguma coisa errada aqui e não deu certo. Vocês testaram? Deu certo?

E23F: Deu certo! Quer fazer? Pode fazer.

Jéssica: Eu quero. Então vamos lá. A gente vai fazer como? Discos e movimentos. [A professora constrói uma tabela (Figura 2) na lousa com duas colunas: discos e movimentos.].

Jéssica: Vamos fazer só para 3, 4 e 5 [discos]. O que é esse $n_{a}$ de vocês?

E23F: Número de movimentos anterior.

Jéssica: $O$ que eu tenho que escrever aqui [aponta pra primeira linha da tabela na coluna movimentos].

E23F: 3 vezes $2+1$.

Jéssica: Esse 3 é o quê?

E33F: Número de movimentos.

E23F: Não, número de discos. 7 é o número de movimentos.

Jéssica: Aqui [na linha abaixo] 4 vezes $2+1$.

E33F: Não professora. Aí é 7 [no lugar do 4 é 7].

Jéssica: Ué! Mas vocês não falaram que era o número de discos?

E23F: Não. Aí é 7!

Jéssica: E porque aqui é 3? [aponta na linha acima para o número 3]

E23F: Porque pra mover 2 [discos] a gente faz 3 movimentos.

Jéssica: Então esse 7 é o 7 daqui. Então 7 x 2+1=15. Aqui coloca 15 e faz 15 x $2+1=31$.

E23F: É!

Jéssica: Caramba! Deu certo! Então poderia ser essa não?

Outro episódio é relativo ao momento em que a professora, ao ser entrevistada por um formador, declara ter sido marcante para ela ser surpreendida com a resposta do grupo $3 \mathrm{~F}$.

Professora: É! E eu não tinha percebido. Na plenária, eu não sei se chega a sistematizar [falando sobre a sua atuação na aula]. Eu não acho que tenha sistematização. Eu acho que ficou o certo e o errado. [...]. [falando sobre o que faltou na aula] A parte de explorar outras soluções. Eu joguei várias vezes antes, mas em 
DOI: $10.20396 /$ zet.v28i0.8659072

nenhum momento eu cheguei em outra solução, e pode ser que exista outra.

O modelo PLOT e a $5^{a}$ TAP (Refletindo sobre a aula)

A $5^{\text {a }}$ TAP foi organizada pelos formadores de modo a valorizar a inclusão de registros de prática (um dos componentes do domínio TAP, no modelo $\mathrm{OAP}$ ), recolhidos na aula desenvolvida na $4^{\text {a }}$ TAP. Os formadores optaram por organizar a $5^{\text {a }}$ TAP (Apêndice II) em torno de vignettes (compostas por protocolos dos estudantes, episódios gravado em vídeo, relatos das observações, entre outros) inseridas em um roteiro com questões para nortear as discussões entre os professores. Nota-se as componentes orquestração e articulação, da dimensão PAF, uma vez que os formadores estavam atentos às interações discursivas que queriam promover entre os participantes, no momento da plenária, assim como, com a intenção de promover a articulação entre os conhecimentos matemáticos e didáticos nos professores participantes. $\mathrm{O}$ desenvolvimento se deu em dois encontros formativos e contou com momentos de (i) discussão em pequenos grupos e (ii) discussão coletiva. Uma escolha importante identificada nas ações dos formadores refere-se à disponibilização, no momento do trabalho em pequenos grupos, de um roteiro e um computador com os episódios da aula. Tal escolha favoreceu que a componente gestão (do PAF) seja mantida na realização da $5^{\text {a }}$ TAP, com os participantes envolvidos em um ambiente de ensino exploratório. No momento da discussão coletiva em plenária, os formadores conduziram e dinamizaram as interações discursivas entre os participantes (IDP), garantido que houvesse espaço e valorização na comunicação dialógica entre todos, bem como favorecesse discussões matemáticas $\boldsymbol{e}$ didáticas de forma articulada. Os formadores utilizaram o mesmo roteiro trabalhado nos pequenos grupos e, coletivamente, assistiram novamente a todos os episódios selecionados.

Por limitação de espaço, no presente artigo restringimo-nos às discussões ocorridas entre os participantes, durante o momento final (plenária), onde depreendemos evidências como os domínios IDP e PAF são contemplados e levam oportunidades de aprendizagem profissional aos professores, em especial, referentes aos conhecimentos didáticos e às ações discursivas.

Mediados e estimulados pela dinâmica implantada pelos formadores, os professores, ao assistirem os episódios, sentiram-se instigados a refletirem e debaterem sobre a atuação da professora. Escolhemos dois momentos para ilustrar esses acontecimentos. Na Figura 7 observamos que os professores exploram as diferentes estratégias matemáticas envolvidas na conversa entre a professora e os alunos, colocando foco nas ações didáticas da professora. Ao fim, eles acabam validando a escolha da professora pelo uso da representação tabular como uma forma de favorecer a organização e a compreensão da generalização encontrada pelos estudantes.

P2 (Professor 2): Eu acho que ficou clara a explicação da estratégia que eles [os alunos] utilizaram porque ela [a professora] foi escrevendo na lousa. Se tivesse sido só uma explicação verbal acho que não teria conseguido entender o raciocínio deles.

P1: É que ela conduz! Eles explicam, mas ela conduz! Essa condução organiza o 
raciocínio.

P4: Eu achei até que na hora que ela deixou eles errarem foi importante também. Porque eles colocaram 3, e eles falaram que era número de discos, e eles mesmos perceberam e corrigiram. Eu achei aquela postura dela importante para eles perceberem o erro que eles estavam cometendo.

Um segundo momento que destacamos está registrado nas reflexões da professora Jéssica em entrevista reflexiva conduzida por um dos formadores ao final da realização da $5^{\text {a }}$ TAP. Observa-se que a professora valoriza a experiência que viveu, ao compartilhar uma aula ministrada por ela com os professores (colegas de formação).

Jéssica: Eu achei que a plenária apontou coisas boas, mas pra mim valeu mais as críticas porque foram críticas construtivas e direcionadas à melhoria da aula. E eu tô tentando mudar algumas práticas em sala de aula depois que eu assisti a minha aula.

$[\ldots]$

Jéssica: Eu aprendi que a preparação da aula é fundamental, mas tão necessária quanto a preparação, é estar pronto para adequar as atividades ao momento. Por vezes eu deixei escapar algumas oportunidades de ensino e de aprendizagem que surgiram no meio das perguntas dos alunos, e isso eu só percebi porque a aula foi gravada e depois eu pude assisti-la. Talvez eu fiquei pensando, talvez deveria ter dado mais oportunidade para algumas perguntas em detrimento de outras. Mas isso eu só vi depois.

\section{Conclusões}

Ainda que se investigue desde longa data o que se entente por "oportunidades de aprendizagem" (Heyd-Metzuyanim, Tabach \& Nachlieli, 2016), foi apenas recentemente que se passou a buscar compreendê-las no âmbito da formação de professores (Tatto \& Senk, 2011). Neste artigo apresentamos um modelo teórico que vem sendo desenvolvido há algum tempo (Ribeiro \& Ponte, 1999), que tem como principais funções possibilitar o design de processos formativos assim como servir ao propósito de guiar os formadores e pesquisadores para compreender se e como ocorrem oportunidades de aprendizagem profissional para os professores participantes em uma formação. Com isso em mente, trouxemos nas seções anteriores algumas situações ilustrativas para indicar o modelo PLOT “em ação" durante todo o processo formativo, desde o momento em que os formadores iniciaram a organização das tarefas de aprendizagem profissional, até o desenvolvimento da formação e consequente identificação de oportunidades para os professores participantes aprenderem sobre padrões, regularidades, generalização e seu ensino quando da abordagem da álgebra na educação básica.

Através da vivência coletiva do Ciclo PDR, foram proporcionados aos professores participantes diferentes momentos de trabalho e reflexão individuais e, especialmente, coletivos, gerando assim oportunidade para que eles aprendessem a partir de "momentos de 
DOI: $10.20396 /$ zet.v28i0.8659072

sala de aula, mas também de planejamento, avaliação e colaboração com colegas e outros" (Davis \& Krajcik, 2005, p. 3). Conjecturamos que isso foi potenciado pela arquitetura do processo formativo, em especial pelo tipo de tarefas disponibilizadas aos professores (Putnam $\&$ Borko, 2000), as quais foram desenhadas e realizadas pelos formadores em um ambiente de ensino-aprendizagem exploratório (Jaworski \& Huang, 2014; Ponte \& Quaresma, 2016), potenciado pela orquestração de discussões didáticas e matemáticas (Stein et al., 2008; Borko, Jacobs, Seago \& Mangram, 2014).

A escolha de uma tarefa matemática de desafio cognitivo elevado (Ponte \& Quaresma, 2016), que possibilitava o surgimento de diferentes padrões matemáticos, na medida em que os alunos realizavam o jogo "Torre de Hanói”, e que levava professor e alunos a pensarem sobre diferentes caminhos para generalização, foi decisivo na e para a qualidade das discussões matemáticas na sala de aula (Ponte \& Quaresma, 2016). Aliado a isso, a organização de um plano de aula amplo e detalhado (Serrazina, 2017) indica o surgimento de uma significativa oportunidade de aprendizagem profissional ligada aos conhecimentos matemáticos sobre padrões e regularidades (Zazkis \& Liljedahl, 2002), bem como a reorganização dos conhecimentos didáticos dos professores sobre o tema (Branco \& Ponte, 2014).

Aos professores foram possibilitadas oportunidades de aprender, a partir da forma como as TAP, em especial a $5^{\mathrm{a}}$, foram organizadas e realizadas pelos formadores. Isso levou os professores participantes a pensarem e refletirem sobre a atuação da professora, a forma como ela conduziu a aula, sendo protagonista em alguns momentos, mas oportunizando esse papel também aos seus alunos. Também foi possível, durante a discussão coletiva na plenária da $5^{\text {a }}$ TAP, perceber que a professora não valorizou o pensamento dos alunos (como identificado nas Figura 5 e 6). No entanto, é de se destacar que as oportunidades de aprendizagem proporcionadas aos professores, devido ao formato do processo formativo, levaram-nos a saírem do isolamento em suas escolas e vivenciarem práticas geradas dentro do grupo, próximas de sua realidade, em especial quando das discussões coletivas que favoreceram interação e aprendizagem uns com os outros (Ball \& Cohen,1999; White et al., 2013).

A experiência de formação considerada neste artigo ilustra os diferentes domínios do modelo OAP, com suas dimensões e componentes, desde a concepção do processo formativo, pelos formadores, até o efetivo desenvolvimento do mesmo. Em especial, destacamos:

- O papel e as ações do formador (PAF) como designers de tarefas formativas, ao optarem por apresentar aos professores, um roteiro para apoiar o planejamento da aula (Serrazina, 2017). Para elaborarem as TAP, os formadores mobilizaram as componentes: articulação entre as dimensões matemática e didática do conhecimento profissional; orquestração de discussões matemáticas e didáticas realizadas ao longo dos encontros formativos; assim como ações do formador no que tange à gestão de um ambiente de ensino-aprendizagem exploratório durante o desenvolvimento das diferentes TAP (alternando momentos de trabalhos em pequenos grupos e em 
plenária).

- As tarefas de aprendizagem profissional (TAP) contemplavam e valorizavam registros de prática, ao trazer para o ambiente formativo, momentos de vivência em salas de aula da educação básica; possibilitavam os professores explorarem uma tarefa matemática para estudantes, tanto do ponto de vista de seus aspectos matemáticos como didáticos, contemplando a componente conhecimento profissional na organização da TAP.

- As interações discursivas entre os participantes (IDP) favoreceram a comunicação dialógica entre todos os professores, e entre estes e o formador, assim como possibilitaram a ocorrência de discussões matemáticas e didáticas de forma articulada.

Com isso, nota-se que o modelo PLOT, tanto possibilitou levantar se e como ocorreram oportunidades de aprendizagem profissional aos professores participantes, discutidas no inicio desta última seção, como foi mobilizado e contemplado durante todas as fases de sua operacionalização (como indicado na Figura 1) no decorrer do processo formativo. Isso corresponde ao que era esperado, tendo em vista que o modelo PLOT foi concebido sob uma perspectiva interativa e interconectada com vistas a atender à "necessidade de desenvolver estruturas compartilhadas para o estudo da aprendizagem dos professores" (Goldsmith, Doerr e Lewis, 2014, p. 23). Naturalmente, a consolidação do modelo PLOT como ferramenta teórico-metodológica para a concepção e desenvolvimento de processos formativos para professores de matemática requer mais estudos.

Nesse sentido, é importante continuar o desenvolvimento do nosso projeto DBR para investigar se e como o modelo PLOT pode ser usado na formação inicial de professores e para identificar de que forma algumas adaptações podem ser necessárias. Também é importante pesquisar a aplicabilidade deste modelo a diferentes conteúdos matemáticos e a outras disciplinas escolares (por exemplo, disciplinas na área das ciências), a fim de melhorar a estrutura do modelo teórico, bem como possibilitar um refinamento e uma abordagem mais ampla e longitudinal.

\section{Agradecimentos}

O artigo foi construído com a colaboração dos autores com outros colegas do grupo de pesquisa ForMatE (Formação Matemática para o Ensino), contando com o financiamento da Fundação de Amparo à Pesquisa do Estado de São Paulo (Fapesp), processo 2018/14.429-2.

\section{Referências}

Adler, J., \& Ronda, E. (2014). An analytical framework for describing teachers' mathematics discourse in instruction. Proceedings of PME 38 and PME-NA 36, 2, 9-16.

Ball, D. L., Ben-Peretz, M., \& Cohen, R. B. (2014). Records of practice and the development of collective professional knowledge. British Journal of Educational Studies, 62(3), 317 335. 
DOI: $10.20396 /$ zet.v28i0.8659072

Ball, D. L., \& Cohen, D. K. (1999). Developing practice, developing practitioners: Toward a practice-based theory of professional education. In G. Sykes \& L. Darling-Hammond (Eds.), Teaching as the learning profession: Handbook of policy and practice (pp. 3-32). San Francisco, CA: Jossey Bass.

Ball, D. L., Thames, M. H., \& Phelps, G. (2008). Content knowledge for teaching: What makes it special? Journal of Teacher Education, 59(5), 389-407.

Beilstein, O. B., Perry, M., \& Bates, M. S. (2017). Prompting meaningful analysis from preservice teachers using elementary mathematics video vignettes. Teaching and Teacher Education, 63, 285-295, http://dx.doi.org/10.1016/j.tate.2017.01.005

Borko, H., Jacobs, J., Eiteljorg, E., \& Pittman, M. E. (2008). Video as a tool for fostering productive discussions in mathematics professional development. Teaching and Teacher Education, 24, 417-436. https://doi.org/10.1016/j.tate.2006.11.012

Borko, H., Jacobs, J., Seago, N., \& Mangram, C. (2014). Facilitating video-based professional development: Planning and orchestrating productive discussions. In Li et al. (Eds.). Transforming mathematics instruction: Multiple approaches and practices (pp. 259-281). DOI 10.1007/978-3-319-04993-9_.

Boston, M., \& Smith, M. (2009). Transforming secondary mathematics teaching: Increasing the cognitive demands of instructional tasks used in teachers' classrooms. Journal for Research in Mathematics Education, 40, 119-156.

Bruce, C. D., Esmonde, I., Ross, J., Dookie, L., \& Beatty, R. (2010). The effects of sustained classroom-embedded teacher professional learning on teacher efficacy and related student achievement. Teaching and Teacher Education, 26, 1598-1608.

Clarke, D., \& Hollingsworth, H. (2002). Elaborating a model of teacher professional growth. Teaching and Teacher Education, 18(8), 947-967.

Cobb, P., Confrey, J., diSessa, A., Lehrer, R., \& Shaube, L. (2003). Designing experiments in educational research. Educational Researcher, 32(1), 9-13.

Cobb, P., Jackson, K., \& Dunlap, C. (2016). Design research: An analysis and critique. In L. English \& D. Kirshner (Eds.), Handbook of international research in mathematics education (pp. 481-503). New York, NY: Routledge.

Coles, A. (2013). Using video for professional development: the role of the discussion facilitator. Journal of Mathematics Teacher Education. 16, 165-184. DOI 10.1007/s10857-012-9225-0

Craig, T., \& Morgan, C. (2015). Language and communication in mathematics education. In S. J. Cho (Ed.), The Proceedings of the $12^{\text {th }}$ International Congress on Mathematical Education, 529-533. DOI 10.1007/978-3-319-12688-3_53

Davis, E. A., \& Krajcik, J. S. (2005). Designing educative curriculum materials to promote teacher learning. Educational Researcher, 34(3), 3-14.

Elias, H. R., Ribeiro, A. J. \& Savioli, A. M. P. (2019). International Journal of Science and Mathematics Education, 18, 357-376. DOI 10.1007/s10763-019-09965-4

Feiman-Nemser, S. (2001). From preparation to practice: Designing a continuum to strengthen and sustain teaching. Teachers College Record, 103(6), 1013-1055. 
DOI: $10.20396 /$ zet.v28i0.8659072

Fuentes, S. Q., \& Ma, J. (2018). Promoting teacher learning: A framework for evaluating the educative features of mathematics curriculum materials. Journal of Mathematics Teacher Education, 21(4), 351-382. https://doi.org/10.1007/s10857-017-9366-2

Goldsmith, L. T., Doerr, H. M., \& Lewis, C. C. (2014). Mathematics teachers' learning: A conceptual framework and synthesis of research. Journal of Mathematics Teacher Education, 17, 5-36.

Heyd-Metzuyanim, E.; Tabach, M., \& Nachlieli, T. (2016). Opportunities for learning given to prospective mathematics teachers: Between ritual and explorative instruction. Journal of Mathematics Teacher Education, 19, 547-574.

Jaworski, B. \& Huang, R. (2014). Teachers and didacticians: Key stakeholders in the processes of developing mathematics teaching. ZDM Mathematics Education 46(2), 173 188. https://doi.org/10.1007/s11858-014-0574-2

Kilpatrick, J. (2019). A double discontinuity and a triple approach: Felix Klein's perspective on mathematics teacher education. In H.-G. Weigand et al. (Eds.), The legacy of Felix Klein, ICME-13 Monographs (pp. 215-225). https://doi.org/10.1007/978-3-319-99386$\underline{7 \_15}$

Lampert, M. (2010). Learning teaching in, from, and for practice: What do we mean? Journal of Teacher Education, 61(1-2), 21-34

Loucks-Horsley, S. (1997). Teacher change, staff development, and systemic change: Reflections from the eye of the paradigm. In S. N. Friel \& G.W. Bright (Eds.), Reflecting on our work: NSF teacher enhancement in K-6 mathematics (pp. 133-150). Lanham, MD: University Press of America.

Maarten, D., den Hertog, J., \& Gravemeijer, K. (2002). Using multimedia cases for educating the primary school mathematics teacher educator: A design study. International Journal of Educational Research, 37(2), 161-178

Mata-Pereira, J., \& Ponte, J. P. (2017). Enhancing students' mathematical reasoning in the classroom: Teacher actions facilitating generalization and justification. Educational Studies in Mathematics, 96(2), 169-186.

Moreira, P. C., \& David, M. M. (2008). Academic mathematics and mathematical knowledge needed in school teaching practice: Some conflicting elements. Journal of Mathematics Teacher Education, 11(1), 23-40. https://doi.org/10.1007/s10857-007-9057-5

Nemirovsky, R., Dimattia, C., Ribeiro, B., \& Lara-Meloy, T. (2005). Talking about teacher episodes. Journal of Mathematics Teacher Education, 8, 363-392.

Neubrand, M. (2018). Conceptualizations of professional knowledge for teachers of mathematics. ZDM Mathematics Education, 50(4), 601-612. https://doi.org/10.1007/s11858-017-0906-0

Ponte, J. P. (1999). Didácticas específicas e construção do conhecimento profissional. In J. Tavares, A. Pereira, A. P. Pedro \& H. A. Sá (Eds.), Investigar e formar em educação: Actas do IV Congresso da SPCE (pp. 59-72). Porto: SPCE.

Ponte, J. P., \& Quaresma, M. (2016). Teachers' professional practice conducting mathematical discussions. Educational Studies in Mathematics, 93(1), 51-66. 
Ponte, J. P., Quaresma, M., Mata-Pereira, J., \& Baptista, M. (2016). O estudo de aula como processo de desenvolvimento profissional de professores de Matemática. Bolema, 30(56), 868-891. doi: 10.1590/1980-4415v30n56a01

Putnam, R., \& Borko, H. (2000). What do new views of knowledge and thinking have to say about research on teacher learning? Educational Researcher, 29(1), 4-15.

Radford L., Barwell R. (2016). Language in Mathematics Education Research. In A. Gutiérrez, G. C. Leder \& P. Boero (Eds) The second handbook of research on the psychology of mathematics education. Rotterdam: Sense.

Remillard, J. T., \& Geist, K. (2002). Supporting teachers' professional learning by navigating openings in the curriculum. Journal of Mathematics Teacher Education, 5, 7-34.

Ribeiro, A. J., \& Ponte, J. P. (2019). Professional learning opportunities in a practice-based teacher education program about the concept of function. Acta Scientiae, 21, 49-74. https://doi.org/10.17648/acta.scientiae.v21iss2id5002

Rowland, T., Huckstep, P., \& Thwaites, A. (2005). Elementary teachers' mathematics subject knowledge: The knowledge quartet and the case of Naomi. Journal of Mathematics Teacher Education, 8(3), 255-281.

Russ, R. S., Sherin, B. L., \& Sherin, M. G. (2016). What constitutes teacher learning? In D. H. Gitomer, \& C. A. Bell (Eds.), Handbook of Research on Teaching (5 ed., pp. 391438). American Educational Research Association.

Serrazina, L. (2017). Planificação do ensino-aprendizagem da Matemática. In GTI (Ed.), A prática dos professores: Planificação e discussão coletiva na sala de aula (pp. 9-32). Lisboa: APM.

Schoenfeld, A. (2015). Thougths on scale. ZDM Mathematics Education, 47(1), 161-169.

Schubring, 2019. Klein's conception of 'elementary mathematics from a higher standpoint'. H.-G. Weigand et al. (Eds.), The legacy of Felix Klein, ICME-13 Monographs (pp. 169180). https://doi.org/10.1007/978-3-319-99386-7_12

Shilo, A., \& Kramarski, B. (2018). Mathematical-metacognitive discourse: How can it be developed among teachers and their students? Empirical evidence from a videotaped lesson and two case studies. ZDM Mathematics Education, 51, 625-640. https://doi.org/10.1007/s11858-018-01016-6.

Silver, E. A., Clark, L. M., Ghousseini, H. N., Charalambous, Y. C., \& Sealy, J. T. (2007) Where is the mathematics? Examining teachers' mathematical learning opportunities in practice-based professional learning tasks. Journal of Mathematics Teacher Education, 10(4-6), 261-277. https://doi.org/10.1007/s10857-007-9039-7

Smith, M. S. (2001). Practice-based professional development for teachers of mathematics. Reston, VA: NCTM.

Smith, M. S., \& Stein, M. K. (1998). Selecting and creating mathematical tasks: From research to practice. Mathematics Teaching in the Middle School, 3, 344-50.

Stein, M. K., Engle, R. A., Smith, M. S., \& Hughes, E. K. (2008). Orchestrating productive mathematical discussions: Five practices for helping teachers move beyond show and tell. Mathematical Thinking and Learning, 10(4), 313-340. https://doi.org/10.1080/10986060802229675 
DOI: $10.20396 /$ zet.v28i0.8659072

Swan, M. (2007). The impact of task based professional development on teachers' practices and beliefs: A design research study. Journal of Mathematics Teacher Education, 10, 217-237.

Tatto, M. T., \& Senk, S. (2011). The mathematics education of future primary and secondary teachers: Methods and findings from the teacher education and development study in mathematics. Journal of Teacher Education, 62(2), 121-137. https://doi.org/10.1177/0022487110391807

Trevisan, A. L., Ribeiro, A. J., \& Ponte, J. P. D. (2020). Professional learning opportunities regarding the concept of function in a practice-based teacher education program. International Electronic Journal of Mathematics Education, 15(2), em0563. https://doi.org/10.29333/iejme/6256

Wasserman, N. (2018). Connecting abstract algebra to secondary mathematics, for secondary mathematics teachers. Cham: Springer.

Webster-Wright, A. (2009) Reframing professional development through understanding authentic professional learning. Review of Educational Research, 79, 702-739. https://doi.org/10.3102/0034654308330970

Zaslavsky, O., \& Leikin, R. (2004). Professional development of mathematics teacher educators: Growth through practice. Journal of Mathematics Teacher Education, 7(4), 532. 